\title{
EL DERECHO FUNDAMENTAL DE ACCESO A LA JUSTICIA DE LOS CONSUMIDORES*
}

\author{
The Fundamental Right of Access \\ to Justice for Consumers
}

\author{
María José Catalán Chamorro \\ Universidad de Valencia (España) \\ maria.jose.catalan@uv.es
}

http://dx.doi.org/10.18543/ed-66(2)-2018pp323-346

Recibido: 30.10.2018

Aceptado: 21.11.2018

\section{Resumen}

El acceso a la justicia es hoy en día un Derecho fundamental reconocido en la Constitución española en el artículo 24 a través del Derecho a la tutela judicial efectiva, así como el derecho a la asistencia justicia gratuita para aquellos que acrediten insuficiencia de recursos para litigar de acuerdo con el artículo 119 de la Constitución española. Sin embargo, estos derechos se desdibujan cuando intentamos reivindicar los derechos de los consumidores. En este trabajo analizamos cómo Cappelletti teorizó, a través de su obra General Report Access to justice, las tres olas básicas para conseguir derribar las barreras que impiden el acceso a la justicia. Esta teoría refleja los pasos seguidos por la Unión Europea para mejorar el acceso a la justicia de los consumidores.

\section{Palabras clave}

Acceso a la justicia; Consumidores; Derecho de consumo; ADR y ODR.

* Cómo citar / Citation 'Chicago-Deusto' (Autor-fecha / Author-date / Lista de referencias / Reference list entries): Catalán Chamorro, María José. 2018. «El Derecho Fundamental de Acceso a la Justicia de los Consumidores». Estudios de Deusto 66, n. ${ }^{\circ}$ : 323-346. http://dx.doi.org/10.18543/ed-66(2)-2018pp323-346. 


\begin{abstract}
The Access to justice is nowadays a fundamental right recognizes by the Spanish Constitution at article 24 throughout the right to obtain the effective protection of the Judges and the Courts as well as the right to Justice shall remain free for those who have insufficient means to litigate according to the article 119 of the Spanish Constitution. However, the blurring of these rights happened when we try to claim consumer's rights. In this paper, we analyze how Cappelletti theorized, through his book General Report Access to justice, the three basic waves for the break down barriers to access to justice. This theory reflects the steps in this implementation taken by the European Union in order to improve the consumer's access to justice
\end{abstract}

\title{
Keywords
}

Access to justice; Consumers; Consumer's rights; ADR and ODR. 


\begin{abstract}
SuMARIO: I INTRODUCCIÓN. II. DESARROLlO DEL DERECHO DE ACCESO A la JUSTICIA EN El ÁMBITO Del CONSUMO. 1. Primer Estadio de Acceso a la Justicia: los colectivos con dificultades en el acceso a la justicia. 1.El acceso a la justicia en Europa. 2. El acceso a la justicia y su relación con la pobreza. 3 Las personas consumidoras en reclamaciones de escasa cuantía. 4. El acceso a la justicia de los consumidores en la Unión Europea. 2. Segundo estadio de Acceso a la Justicia: representación de los intereses difusos y colectivos de los consumidores. 1. Ámbito nacional. 2. Ámbito Europeo. 3. El tercer estadio de Acceso a la Justicia: resolución alternativa de conflictos en consumo. 4. Cuarto estadio de acceso a la justicia: Resolución alternativa de conflictos de consumo en línea.
\end{abstract}

\title{
I. INTRODUCCIÓN
}

El derecho de acceso a la justicia es un derecho reconocido de una u otra manera en todas las constituciones contemporáneas, así mismo no nos cabe duda de su realidad en España gracias a la Ley 1/1996, de 10 de enero, de asistencia jurídica gratuita. ${ }^{1}$ Sin embargo, este derecho queda desdibujado cuando se trata de reclamaciones de escasa cuantía, fundamentalmente derivadas de la adquisición de bienes o servicios por parte de los ciudadanos consumidores y cuya entidad no es suficiente para ser reclamada a través de un largo y tedioso proceso judicial, con aportación de pruebas y donde la quota litis será muy superior a la cantidad reclamada.

Este difícil acceso a la justicia para los consumidores se agudiza hoy más que nunca con las compras a través de internet a otros países fundamentalmente de la Unión Europea, así como con la inclusión de productos contratados sin un consentimiento suficiente y debidamente informado en los servicios de suministros, telecomunicaciones o bancarios. A todo ello, le unimos el alto porcentaje de consumidores vulnerables existentes en nuestro país, donde ronda el $39 \%$ de la población ${ }^{2}$ dándonos todo esto una ecuación de alto riesgo para los intereses económicos de las ya delicadas economías domésticas ${ }^{3}$.

${ }^{1}$ BOE núm. 11, de 12 de enero de 1996.

2 Consumer vulnerability across key markets in the European Union (Final Report), Ed Publications Office of the European Union, enero 2016. Figure 39. Level of vulnerability on average across all dimensions, by country group from the approach-based typology, p. 380.

${ }_{3}$ No obstante, la percepción ciudadana es más optimista, según el último Eurobarometro sobre opinión publica en la Unión Europea, los ciudadanos encuentran mejoras en sus compras de bienes y servicios a otros países de la UE, hasta 13 puntos por encima de 
Por ello, siguiendo la estela marcada por Cappelletti en su obra Acces to Justice, la Unión Europea ha intentado superar las barreras que Cappelletti teorizó en el acceso a la justicia. En primer lugar, la superación de la pobreza con la asistencia jurídica gratuita, en segundo lugar, la superación de las reclamaciones masivas con las acciones colectivas y, en tercer lugar, la facilitación del acceso a una tutela efectiva a través de los mecanismos de resolución alternativa de conflictos. Hoy, podríamos evolucionar el acceso a la justicia a través estos mecanismos de resolución alternativa en su esfera online.

No obstante, aún queda mucha tarea por hacer en cada una de las tres esferas teorizadas por Cappelletti, tanto en el ámbito de la pobreza, como en las dificultades para hacer realidad las acciones colectivas y en el avance de los ADR y los ODR para solventar los conflictos que no precisen de un proceso judicial en toda su extensión.

\section{DESARROLLO DEL DERECHO DE ACCESO A LA JUSTICIA EN EL ÁMBITO DEL CONSUMO}

Los orígenes del «acceso a la justicia» fueron alegados por primera vez ante la Corte Suprema de Estados Unidos, en el caso Lessee of Pollard's Heirs v. Kibbe 39 U.S. 353, en 1840, en virtud del Tratado de Amistad, Límites y Navegación de $1795^{4}$. Sin embargo, este principio no sería desarrollado con la entidad suficiente ${ }^{5}$ tanto en las legislaciones como en los estudios de

cómo se encontraban en 2014. Standard Eurobarometer 89 Public opinion in the European Union (marzo 2018).

${ }^{4}$ También denominado como Tratado de San Lorenzo en España o Tratado Pinckney en Estados Unidos, firmado entre estos dos países con el objetivo de definir las fronteras entre Estados Unidos y las colonias españolas en Norteamerica, regular los derechos de navegación en el rio Misisipi, acuerdos de paz, libertad de comercio y libertad de acceso a la justicia para los ciudadanos, concretamente en el artículo XX de la declaración se estableció: «Se ha convenido igualmente que los habitantes de los territorios de una y otra parte respectivamente serán admitidos en los Tribunales de justicia de la otra parte, y les será permitido el entablar sus pleitos para el recobro de sus propiedades, pago de sus deudas y satisfacción de los daños; bien sean las personas contra las cuales se quejasen súbditos o ciudadanos del país en que se hallen; o bien sean cualquiera otros sujetos que se hayan refugiado alli. Y los pleitos y sentencias de dichos Tribunales serán las mismas que hubieran sido en el caso de que las partes litigantes fuesen súbditos o ciudadanos del mismo pais.».

5 Podemos encontrar algunas referencias bibliográficas al respecto, tras la Segunda Guerra Mundial, cuando los juristas comienzan a ser conscientes de la necesidad de mejorar el acceso a la justicia de la ciudadanía. Como: William T., Gosset, «Access to Justice: The True Significance of Legal Aid», American Bar Association Journal 40, (1954), pp. 111-112 y Orison S., Marden, «Equal Access to Justice: The Challenge and the Op- 
Derecho, hasta el nacimiento del Proyecto de «Acceso A La Justicia» de referencia en la mayoría de estudios del campo, realizado por Cappelletti junto con Garth en la Universidad de Florencia en la década de los setenta ${ }^{6}$.

Esta obra nos servirá de modelo en el presente trabajo para comparar y analizar las tres «olas de reformas» teóricas que ha tenido el movimiento de acceso a la justicia, con los movimientos legislativos por parte de la Unión Europea, y por ende también de España, para facilitar el acceso a la justicia de los consumidores ${ }^{7}$.

La primera «ola» vendrá caracterizada por el reconocimiento de la asistencia jurídica gratuita para las personas sin recursos suficientes como para acceder a la justicia por sus propios medios.

La segunda «ola» se fundamenta en el movimiento de representación de los intereses difusos e intereses colectivos ${ }^{8}$, mediante los mecanismos de las acciones colectivas, de los operadores del Derecho en defensa del interés público y el reconocimiento de capacidad procesal para demandar a los grupos o asociaciones de consumidores o en defensa del medioambiente.

Finalmente, estas dos «olas» no funcionarían tan solo con la inclusión de estos derechos en provisiones legales. Para ello, la tercera «ola» incluye y absorbe las otras dos. En esta ocasión el protagonista es el abanico de procedimientos e instituciones que comprende la maquinaria del Alternative Dispute Resolution (en adelante, ADR). Desde esta perspectiva, se apunta de forma renovada a un procedimiento de resolución de conflictos más informal para las reclamaciones de escasa cuantía que, hasta el momento, solo podrían dirimir los tribunales de justicia ${ }^{9}$. Incluso, por primera vez, se pensó en la

portunity», Wash. \& Lee L. Rev., No 19, (1962), pp.153-164. Ambas son prácticamente las únicas referencias bibliográficas que aparecen con la idea de acceso a la justicia antes de los años setenta.

${ }^{6}$ Mauro Cappelletti y Bryant G. Garth, Access to Justice: A World Survey, Vol I., Libro 1, Italia, (1978). Sijthofff and nooordhoff- Alphena Andenrijn. Dott. A. Giufrè Editore-Milan, pp. 22-54.

7 Ver mas en: Thierry Bourgoignie, «L'accès des consommateurs au droit et à la justice: les dé s du marché unique», REDC, (1992), pp. 124- 125 y Geraint G Howells y Stephen Weatherill, Consumer Protection Law, Ashgate, Londres, (2005) (2a ed.), pp. 651-652.

${ }^{8}$ Ver mas en: Christopher Hodges, The reform of Class and Representative Actions in European Legal Systems. A new framework for collective redress in Europe», Hart Publising, Oxford, (2008), pp.185-191 y Stefan Wrbka, Steven Van Uytsel, Mathias M. Siems., "Access to Justice and Collective actions: «Florence and beyond», Collective actions: enhancing access to justice and reconciling multilayer interest,? Ed. Stefan Wrbka, Steven Van Uytsel, Mathias M. Siems., Cambridge University Press, United State of America, (2012), pp. 8-10.

9 Según el Eurobarómetro 397 de septiembre de (2015) sobre «Consumer attitudes towards cross-border trade and consumer protection», solo el $76 \%$ de los consumidores que tuvieron un caso de reclamación ejercitaron algún tipo de acción para solventarla, y 
reorganización del sistema, a través de servicios de asistencia legal mediante asistentes legales o paralegal, fuera de la organización del Poder Judicial.

Esta perspectiva acarrea la preocupación por los riesgos provenientes de simplificar en exceso los mecanismos de resolución de conflictos, amenazando la protección esencial del procedimiento. Sin embargo, el estudio del presente trabajo se asienta en el convencimiento de que es necesario apostar y arriesgarse a realizar un sistema que mejore las oportunidades de acceso a la justicia, para que los ciudadanos, individual o colectivamente, como consumidores de bienes y servicios puedan exigir y reivindicar sus derechos ante los abusos del mercado ${ }^{10}$. No obstante, se tendrá que vigilar que se realice con todas las garantías procesales que ofrece nuestro Estado de Derecho.

Estas exigencias se han visto respondidas mediante la creación de vías para facilitar al ciudadano el acceso a la justicia o, al menos, dotarlo de una tutela judicial efectiva mínima para evitar la merma de derechos de los ciudadanos como consumidores del mercado único europeo. Todo ello acompañado del ejercicio de una discriminación positiva ${ }^{11}$ hacia la parte más débil de las relaciones jurídicas comerciales, es decir, los consumidores, ante los abusos del mercado ${ }^{12}$.

\section{Primer Estadio de Acceso a la Justicia: los colectivos con dificultades en el acceso a la justicia}

Siguiendo la obra de Cappelletti y los pasos de la Unión Europea a lo largo de su historia, podemos relacionar prima facie el acceso a la justicia con

de ellos, solo un 3\% lo ejercieron ante los tribunales de justicia. Por otro lado, hemos de destacar que solo un 7\% eligió un sistema ADR mientras que un $83 \%$ prefirieron reclamar directamente al comerciante, un $18 \%$ al fabricante y un $8 \%$ a una autoridad pública. Sin embargo, a pesar esas cifras, los más satisfechos con la forma de resolución de los conflictos fueron los resueltos a través de un ADR en un $68 \%$, seguidos por los que reclamaron al comerciante con un $60 \%$ de satisfacción y los que se dirigieron al fabricante o a una autoridad pública se mostraron satisfechos en un $59 \%$ de los casos. Hemos de denotar como dato característico que los que acudieron a los tribunales ordinarios de justicia estaban satisfechos tan solo en un $44 \%$, la calificación más baja.

${ }^{10}$ Mauro Cappelletti y Bryant G. Garth, Access to Justice: A World Survey, op. cit. pp. x y xi.

${ }^{11}$ Luis Prieto Sanchís, «Los derechos sociales y el principio de igualdad sustancial», $R e$ vista del centro de estudios constitucionales, No 22 (septiembre-diciembre) (1995), pp. 9-57.

12 Parte de la doctrina se ha mostrado en contra de esta discriminación positiva, debido a que en ocasiones podemos denotar como parte débil a algunos comerciantes ante consumidores que han abusado o abusan del sistema de protección de los derechos de los consumidores. Ewoud H. Hondius, «The Protection of the Weak Party in a Harmonised European Contract Law: A Synthesis», Journal of Consumer Policy, September (2004), Vol. 27, Issue 3, pp 245-251. 
la pobreza, las reclamaciones de escasa cuantía y su importancia en el ámbito del consumo.

\subsection{El acceso a la justicia en Europa}

La Unión Europea se inició en el reconocimiento del Derecho de acceso a la justicia de los ciudadanos en el Convenio para la Protección de los Derechos Humanos y de las Libertades Fundamentales ${ }^{13}$, adoptado en Roma el 4 de noviembre de 1950 por el Consejo de Europa ${ }^{14}$. En este Convenio podemos apreciar un primer estadio mediante el reconocimiento de acceso a la justicia de los colectivos más desfavorecidos y/o con dificultades económicas. En el artículo 6 se reconoce precisamente el derecho a un proceso equitativo, así como en el artículo 13 el derecho a un recurso efectivo. Ambos derechos son esenciales para el acceso a la justicia de todos los consumidores que sientan haber sido vulnerados en sus derechos en un plano de igualdad material con los comerciantes. Sin embargo, en nada se pronunciaba respecto de las personas sin recursos y cómo estas podrían acceder a la justicia a través de una representación y asistencia gratuitas. En el mismo sentido camina la Carta de los Derechos Fundamentales de la Unión Europea, proclamada formalmente en Niza en diciembre del año 2000 por el Parlamento Europeo, el Consejo y la Comisión ${ }^{15}$, reconociendo en su artículo 47 el derecho a la tutela judicial efectiva, a un juez imparcial y a la asistencia jurídica gratuita.

Es obligado apuntar que la Constitución Española de 1931 fue unas de las primeras en Europa en garantizar el acceso gratuito a la justicia de todos los ciudadanos que lo necesitasen por motivos económicos en su artículo $94^{16}$. En Alemania, entre 1919 y 1923, se puso en marcha, como un derecho fundamental de la ciudadanía, un sistema estatal de compensación a través de abogados privados ${ }^{17}$. Posteriormente, la Constitución francesa de 1946 exigió una justicia efectiva y accesible a todos en su artículo 17, artículo ampliado en su reforma de 1958. En Inglaterra no sería hasta 1949 cuando de una forma más decidida acometiesen la reforma definitiva, creando el Estatuto sobre

${ }^{13}$ Comúnmente conocido como Convención Europea de Derechos Humanos, e inspirada en Declaración Universal de los Derechos Humanos de 1948.

14 Posteriormente enmendado por los Protocolos adicionales números 3 y 5 , de 6 de mayo de 1963 y 20 de enero de 1966, respectivamente. Y ratificado por España en BOE núm. 243, de 10 de octubre de 1979, pp. 23564 a 23570.

15 Carta de los Derechos Fundamentales (2000/C 364/01).

16 Disponible en: http://www.congreso.es/docu/constituciones/1931/1931_cd.pdf, acceso 11 de octubre de 2018.

${ }^{17}$ La Ley fundamental alemana de 1949 que reconoció el derecho a un juez natural. 
Asesoramiento y Asistencia Legal ${ }^{18}$, sistema de asesoramiento gratuito proporcionado por The Law Society, la asociación nacional de abogados.

De esta manera se empezaba a construir el Estado Social, nuevos derechos como consumidores, como trabajadores, como propietarios, en definitiva, como ciudadanos en igualdad de condiciones. Era el momento de reconocer el derecho de acceso a las técnicas procesales que garantizasen la función de servicio social de los tribunales. En la actualidad, casi todos los Estados miembros reconocen en sus constituciones el Derecho de acceso a la justicia, aunque no todos lo contemplan de la misma manera ${ }^{19}$. El artículo 18 (2) de la Constitución holandesa garantiza el acceso a la asistencia jurídica a personas con medios limitados, el artículo 24 de la Constitución italiana reconoce el acceso a la justicia y la asistencia jurídica gratuita para aquellos ciudadanos que la precisen por motivos económicos y en el mismo sentido lo hace la Constitución española en su artículo 119 sobre el acceso a la justicia gratuita $^{20}$.

El derecho de acceso a la justicia se ha convertido en un derecho social que ha ido evolucionando a lo largo del tiempo y que juega un papel esencial en cualquier Estado democrático y de Derecho del mundo. En primer lugar, como veremos, se lucha por un acceso a la justicia que podríamos calificar de simplificado ${ }^{21} \mathrm{y}$, a posteriori, hacia un derecho de acceso a la justicia de los consumidores, como derecho fundamental de segunda generación ${ }^{22}$ pero sin perder de vista que las dificultades para acceder a la justicia por reclamaciones

${ }_{18}$ Legal Aid and Advice Act 1949, esta fue creada tras la publicación del Rushcliffe Committee Report en 1945, donde se establecían principios elementales para el acceso a la justicia de las personas más vulnerables. «Submission of the Law society of England and Wales to the Labour Party Review of Legal Aid», The Law Society. Febrero 2016, p. 2.

19 Alemania (Artículo 101), Austria (Artículo 83), Bélgica (Artículo 13 of Capitulo II), Luxemburgo (Artículo 13), República Checa (Artículo 38), o España (Artículo 24), Finlandia (artículo 21), Italia (artículo 24) Eva Storskrubb y Jacques Ziller, «Access to Justice in European Comparative Law», Access to Justice as a Human Right, Coord. Francesco Francioni, Oxford University Press, Oxford, (2007), pp. 188-191.

${ }^{20}$ Así como el artículo 52.3 de la Constitución británica donde se limitan las tasas prevaleciendo el Derecho de acceso a la justicia, artículo 21 de la Constitución rumana.

${ }^{21}$ Elvira Méndez Pinedo, La protección de Consumidores en la Unión Europea, hacia un Derecho Procesal comunitario de consumo, Marcial Pons, Ediciones jurídicas S.A., Madrid, (1998), pp. 164-168 y 237.

${ }_{22}$ Encarnación Fernández Ruiz-Gálvez, «Los derechos económicos, sociales, culturales, V. Los derechos humanos de segunda y tercera generación», Manual de Derechos Humanos, Coord. José Justo Megías Quirós, Thomson Aranzadi, 2006, pp. 103-137; Victor Abramovich y Christian Courtis, «La estructura de los derechos sociales en el problema de su exigibilidad», Los derechos sociales como derechos exigibles, Trotta, Madrid, (2002), pp. 19-20 y 36, y José María Rodríguez Paniagua, Moralidad, derechos, valores, Civitas, Madrid, (2003), pp. 111-122. 
de consumo de escasa cuantía afecta de forma dramática a las economías domésticas, hoy más débiles que nunca por los efectos aun latentes de la crisis económica.

El obstáculo más evidente y primigenio al que debemos hacer frente es la pobreza. Y no solo la pobreza como falta de capacidad económica, sino también la devenida de circunstancias concomitantes que rodean a esta situación como las dificultades de acceso a la información, a la formación para conocer sus derechos o al desconocimiento sobre cómo o dónde obtener representación o asistencia legal ${ }^{23}$.

En el sector privado, sobre todo en el sector de las compañías aseguradoras, han proliferado con bastante éxito los seguros de defensa jurídica, debido a la creciente necesidad de este tipo de productos en las últimas décadas. Sin embargo, en España han tenido menos éxito que en el resto de Europa debido a la cobertura que la Ley 1/1996, de 10 de enero, de asistencia jurídica gratuita presta a las personas con dificultades -no solamente económicas sino también sociales-, para el acceso a la justicia en igualdad de condiciones que el resto de ciudadanos. Esta tipología de seguro fue regulada, en España, por imperativo de norma europea, en concreto por la Directiva 88/357/CEE, sobre libertad de servicios en seguros distintos al de vida, y de actualización de la legislación de seguros privados a través de la Ley 21/1990, de 19 de diciembre, para adaptar el Derecho español a la Directiva 87/344/CEE, de 22 de junio, sobre coordinación de las disposiciones legales, reglamentarias y administrativas relativas al seguro de defensa jurídica ${ }^{24}$, en su sección novena ${ }^{25}$ donde, de forma poco acertada, fue incluida como un seguro de daños, ya que la naturaleza de este seguro de daños dicta mucho de la finalidad del seguro de defensa jurídica. Por un lado, no es aplicable el principio indemnizatorio, ni la mayoría de los preceptos del seguro de daños. Por otro lado, la regulación sobre la prestación de un servicio de asistencia jurídica por el asegurador, queda regulado fuera del seguro de daños. Por ello, es defendido por la mayoría de la

${ }^{23}$ Mauro Cappelletti, «Alternative Dispute Resolution Process within the Framework of the worldwide Access to justice Movement» Toward Equal Justice: A Comparative Study of Legal Aid Modern Societies, Vol. 56, (1993), pp. 282-296 y Mauro Cappelletti, James Gordley y Earl Johnson, Toward Equal Justice: A Comparative Study of Legal Aid Modern Societies, Milan \& Dobbs Ferry, New York, (1975), pp. 343-353.

${ }^{24}$ Modificada posteriormente en lo que respecta al seguro de defensa jurídica por la Directiva 2009/138/CE del Parlamento Europeo y del Consejo, de 25 de noviembre de 2009, sobre el seguro de vida, el acceso a la actividad de seguro y de reaseguro y su ejercicio (Solvencia II) a través de la Ley 20/2015, de 14 de julio, de ordenación, supervisión y solvencia de las entidades aseguradoras y reaseguradoras.

${ }^{25}$ BOE núm. 304, de 20/12/1990. 
doctrina como una figura independiente, de naturaleza compleja y sin claro encuadre sistemático ${ }^{26}$.

\subsection{El acceso a la justicia y su relación con la pobreza}

El concepto de pobreza ha evolucionado a lo largo de las últimas décadas con gran intensidad, debido al auge del Estado Social y de Derecho. La concepción de la pobreza en los años setenta cuando Cappelletti pone por primera vez el foco de atención en este sector de la población altamente vulnerable ante las dificultades de acceso a la justicia hoy debe ser actualizado y contextualizado, analizando de manera transversal a través de diferentes prismas, como los factores sociológicos. Es decir, estudiando todas las circunstancias concomitantes que agravan o pueden agravar las dificultades de acceso a la justicia, amén de tener en cuenta siempre el elemento común denominador a todos ellos, la pobreza o la exclusión social, como obstáculo principal para acceder en plenitud e igualdad de condiciones a la justicia, en el caso que nos ocupa en el presente trabajo, de los consumidores.

El artículo 9.2 de la Constitución española (en adelante CE), exige a los poderes públicos que promuevan las condiciones para que la libertad y la igualdad del individuo y de los grupos en que se integran sea reales y efectivas, así como a remover los obstáculos que impidan o dificulten su plenitud. Estos obstáculos tienen su origen en diferentes ámbitos como el económico, cultural, racial, sexual, religioso, de género, psicológico o idiomático ${ }^{27}$. Todo ello, unido al artículo $24 \mathrm{CE}$ sobre el derecho a la tutela judicial efectiva y al artículo $51 \mathrm{CE}$ que garantiza la defensa de los legítimos intereses económicos de los consumidores y usuarios por parte de los poderes públicos, hacen que podamos sentirnos constitucionalmente protegidos como consumidores en nuestro país.

Sin embargo, las estadísticas nos dicen que aún nos encontramos lejos de copar estos objetivos en lo que a la justicia social se refiere con respecto a los consumidores y usuarios. Así las cosas, aunque el conocimiento de los derechos de los consumidores haya crecido en los últimos años en la Unión Europea, España, en el año 2017 se sigue encontrando 1,4 puntos por debajo de la media, siendo uno de los seis países miembros que más decrecen en el

${ }^{26}$ Juan Carlos Velasco Perdigones, «El Seguro de Defensa Jurídica: problemas doctrinales e interpretativos actuales», Diario La Ley, No 8653, (2015), Sección Doctrina, 25 de noviembre de (2015).

27 Marc Galanter, «Access to Justice in a World of Expanding Social Capability», Fordham Urban Law Journal, Vol. 37, Issue 1, (2009), pp. 115-128. 
conocimiento de sus derechos ${ }^{28}$. A este dato se le une el hecho de que nuestro país sea considerado el segundo de la Unión Europea más expuesto a prácticas comerciales injustas o desleales, solo por detrás de $\mathrm{Croacia}^{29}$. A pesar de estos malos datos somos el país mejor valorado para comprar online $^{30}$, es decir, cuando actuamos como vendedores cumplimos con la legalidad vigente, pero cuando actuamos como compradores no conocemos nuestros derechos y por ende estamos más expuestos a que se nos vulneren los mismos.

Asimismo, según los últimos datos disponibles el problema del acceso a la justicia de los consumidores no ha sido suficientemente superado, el Marcador de Justicia alerta sobre los serios problemas de acceso a la justicia que existen en la Unión Europea en relación especialmente con la pobreza y los consumidores, es decir, consumidores pobres que no reciben ningún tipo de asistencia ni ayuda ante practicas injustas por parte de las empresas ${ }^{31}$. Mientras la mayoría de países de la Unión Europea han endurecido las condiciones para el acceso a la asistencia jurídica gratuita en los últimos años, los niveles de pobreza no han parado de aumentar ${ }^{32}$.

Así las cosas, debemos apuntar que no solo ha cambiado el concepto de pobreza, sino que han surgido cambios en torno al concepto de acceso a la justicia. En la época liberal, a finales del Siglo XVIII y principios del XIX, el proceso civil reflejaba la filosofía del individualismo más absoluto y donde la justicia, como cualquier otro producto del sistema laissez faire, podía ser comprada solo por aquellos que pudiesen pagar su coste, debido a que las personas eran consideradas las únicas responsables de su destino ${ }^{33}$. En el Siglo XX el reconocimiento de los derechos y libertades de forma internacional se asienta, y no meramente como declaración programática sino inspirador de enormes cambios jurídicos y estructurales. Sin embargo, como se puso de manifiesto en la primera parte del Siglo XX las desigualdades económicas y sociales generaban una palmaria desigualdad en el marco de la resolución de conflictos, o de la obtención de Justicia. Esto fue en gran medida uno de los elementos que propiciaron la aparición del movimiento libre de acceso a cualquier medio de tutela o de Justicia, esto es, también la gente más

${ }^{28}$ Último Consumer Conditions Scoreboard, (2017), p. 17. Disponible en: http://ec. europa.eu/newsroom/just/item-detail.cfm?item_id=117250, acceso el día 11 de octubre de 2018.

29 Ibidem, p. 41.

30 Ibidem, p. 101.

31 The 2018 EU Justice Scoreboard, pp. 32 y 33, disiponible en: https://ec.europa.eu/ info/sites/info/files/justice_scoreboard_2018_en.pdf, acceso el día 11 de octubre de 2018.

32 Íbidem p. 47.

${ }^{33}$ Mauro Cappelletti y Bryant G. Garth, «General Report», Access to Justice, Vol I., Book 1, op. cit. p. 7. 
desfavorecida económicamente empezaba a tener acceso a través de la mediación a cauces de tutela que no eran los tribunales ordinarios de justicia, estructurados y desarrollados gracias a la enorme dosis de generosidad y voluntariedad de los movimientos sociales inspirados en el realismo jurídico, la búsqueda de la paz social ${ }^{34}$. Fue entonces cuando junto con el realismo jurídico nace la comentada obra de Cappelletti.

Así las cosas, el derecho de acceso a la justicia existía formalmente desde las proclamaciones legislativas anteriormente comentadas, sin embargo, las diferencias sociales y económicas hacían de éste un derecho inaplicable para la gran mayoría de la población. Para paliar esta situación fueron surgiendo movimientos en defensa de los derechos y deberes sociales desde los gobiernos, de las comunidades, asociaciones y particulares para con la sociedad ${ }^{35}$.

En el desarrollo de nuestra investigación, reflejaremos cómo esta tendencia ascendente en la facilitación del acceso a la justicia puede estar decreciendo especialmente en el ámbito de la justicia de consumo, debido a la actual concepción del sistema capitalista globalizado que supedita a los consumidores y sus derechos a los intereses de grandes compañías ${ }^{36}$. Unidos estos intereses económicos, a las innovaciones del big data y los medios de resolución alternativos de justicia online y offline podríamos fácilmente pensar que el acceso a la justicia para los consumidores es hoy más fácil que antaño. Esta prima facie es correcta, sin embargo, debemos tener presente que son estos mismos intereses económicos en forma de empresas o macroempresas que vulneran nuestros derechos, los que nos ofrecen a posteriori estos métodos de resolución alternativa de los conflictos que ellos mismos han creado a través de figuras como el defensor del cliente. Figuras que, aunque organizativamente aparecen como independientes de la empresa, son nombradas por sus consejos de administración amén de regir estos sus actividades.

${ }^{34}$ Silvia Barona Vilar, Solución extrajurisdiccional de conflictos: Alternative dispute resolution (ADR) y derecho procesal, Tirant Lo Blanch, Valencia, (1999), pp. 34-44.

${ }_{35}$ Mauro Cappelletti, Processo E Ideologie, Bolonia II Mulino, (1969), pp. 511-524 y Mauro Cappelletti y Bryant G. Garth, «La cambiante concepción teórica del acceso a la justicia» $\mathrm{y}$ «Enfoques prácticos a los problemas de acceso a la justicia», El acceso a la justicia: la tendencia en el movimiento mundial para hacer efectivos los derechos, traducción de Mónica Miranda, Fondo de Cultura Económica, México, (1996) pp. 11-13 y 2425 .

36 Juan Ignacio Font Galan, «Apogeo de racionalidad económica y disenso constitucional. Prólogo para un alegato en defensa del constitucionalismo social y del deber jurídico-público de protección de los consumidores» La protección de los consumidores en tiempos de cambio: ponencias y comunicaciones del XIII Congreso de la Asociación Sainz de Andino, coord., Javier Pagador López, Manuel Pino Abad, Dir. Luis María Miranda Serrano, Iustel, Madrid, (2015), pp. 589-620. 


\subsection{Las personas consumidoras en reclamaciones de escasa cuantía}

Volviendo al camino recorrido por la Unión Europea poco a poco surgió la necesidad de reconocer el derecho de acceso a la justicia y la protección de los consumidores que actuaban en el Mercado Único de la Unión Europea, en los inicios del Derecho de Consumo, siguiendo la experiencia del laissez faire. En un primer momento, los países miembros se pusieron de acuerdo para acometer un reconocimiento formal de los Derechos fundamentales de los consumidores -a través de múltiples comunicaciones, informes, decisiones, resoluciones, dictámenes, recomendaciones, libros verdes, conferencias, simposios, comités de expertos etc., desde $1975^{37}$-, pero sin las herramientas procesales necesarias para ejercerlos de forma efectiva, hasta prácticamente la entrada en vigor de la Directiva 2013/11/UE sobre ADR de consumo y el Reglamento 524/2013 sobre la plataforma de ODR. Instrumentos legislativos que también adolecen de multitud de mermas en lo referente a las garantías que ofrecen para asegurar la tutela efectiva a las personas consumidoras, pero que se plantea en la actualidad como única tabla de salvación para este acceso a la justicia.

En la búsqueda de la efectividad de este acceso a la justicia debemos señalar, en primer lugar, los costes del procedimiento en general. En concreto, cabe prestar especial atención a la agudización de esta dificultad, especialmente cuando hablamos de reclamaciones de escasa cuantía, debido sobre todo debido a los costes procesales de las mismas, pues son porcentualmente muy superiores a las reclamaciones de mayor cuantía ${ }^{38}$. En otras palabras, si los costes procesales de un procedimiento de escasa cuantía ostentan cuantitativamente un mayor porcentaje sobre el valor reclamado, los ciudadanos no podrán asumir el coste que esto representa sobre el total de la reclamación. Para eliminar esta barrera, ya vislumbrada por Cappelletti en los años setenta, la Unión Europea, en su voluntad de mejorar las condiciones de los consumidores y grupos de consumidores que realizan compras transnacionales en el Mercado Único Europeo, creó el proceso europeo de escasa

${ }^{37}$ Con el Coloquio sobre los medios judiciales y cuasi-judiciales para la protección de los consumidores, que la Comisión organizó en 1975 ver más en: María José Catalán Chamorro, El acceso a la justicia de los consumidores a través del ADR y del ODR, Tirant Lo Blanch, Valencia, (2018), p. 52.

38 En este sentido, cuatro de cada diez consumidores con una causa legítima para reclamar no toman ninguna acción al respecto, un $40 \%$ no creen en poder obtener una solución satisfactoria, un $38 \%$ creen que conlleva demasiado tiempo, mientras que un $34 \%$ creen que la reclamación es de demasiado poco valor. según el Eurobarómetro 397 de septiembre de 2015 sobre «Consumer attitudes towards cross-border trade. and consumer protection», p. 13. 
cuantía ${ }^{39}$. Este proceso reduce los costes de las reclamaciones de una cuantía, en materia civil o mercantil, inferior a $5.000 \operatorname{euros}^{40}$. Sin embargo, no los elimina, pues en la mayoría de países europeos existe una tasa por admitir una demanda en este proceso europeo, tramitándose la demanda solo previo abono de la tasa ${ }^{41}$.

Hay que tener en cuenta aspectos como la capacidad económica de las partes, en cuanto a sus recursos económicos ${ }^{42}$ así como su capacidad de obrar, competencias en formación, situación social ${ }^{43}$, amén de la posición desigual causada por el desconocimiento del procedimiento de reclamación de consumo por parte del consumidor, a diferencia del empresario que

${ }^{39}$ Reglamento (CE) n 861/2007 del Parlamento Europeo y del Consejo, de 11 de julio de 2007, por el que se establece un Proceso Europeo De Escasa Cuantía.

Este reglamento ha sido modificado en algunos de sus puntos por el Reglamento (UE) 2015/2421 del Parlamento Europeo y del Consejo de 16 de diciembre de 2015 por el que se modifican el Reglamento (CE) $n^{\circ} 861 / 2007$ por el que se establece un proceso europeo de escasa cuantía, y el Reglamento (CE) $n^{\circ} 1896 / 2006$ por el que se establece un proceso monitorio europeo.

Los orígenes de este Reglamento comienzan el 30 de noviembre de 2000, el Consejo adoptó un programa conjunto de la Comisión y del Consejo de medidas para la aplicación del principio de reconocimiento mutuo de las resoluciones judiciales en materia civil y mercantil. Posteriormente, declarado este como el Programa de La Haya, adoptado por el Consejo Europeo el 5 de noviembre de 2004, se abogaba por que se prosiguieran activamente los trabajos relativos al proceso de escasa cuantía. Finalmente, el 20 de diciembre de 2002, la Comisión Europea aprobó el Libro Verde sobre el proceso monitorio europeo y las medidas para simplificar y acelerar los litigios de escasa cuantía.

${ }^{40}$ El Reglamento (UE) 2015/2421 del Parlamento Europeo y del Consejo de 16 de diciembre de 2015 por el que se modifican el Reglamento (CE) $n^{\circ} 861 / 2007$ por el que se establece un proceso europeo de escasa cuantía, y el Reglamento (CE) no 1896/2006 por el que se establece un proceso monitorio europeo, prevé en su artículo 2, la elevación del límite relativo a la cuantía hasta los 5.000 euros, con el fin de mejorar el acceso a la tutela judicial efectiva y económicamente eficiente en los litigios transfronterizos. Así como reforzar la confianza en las transacciones transfronterizas y que contribuirá positivamente al aprovechamiento máximo de las oportunidades que ofrece el mercado interior europeo. Esta modificación es aplicable desde el 14 de julio de 2017.

${ }^{41}$ Guía Práctica para la aplicación del Proceso Europeo de Escasa Cuantía, con arreglo al Reglamento (CE) no 861/2007 del Parlamento Europeo y del Consejo, de 11 de julio de (2007), por el que se establece un Proceso Europeo De Escasa Cuantía, publicado por Unión Europea, (2013), p. 34.

42 Eurobarómetro 395 de abril de 2013 «sobre reclamaciones de escasa cuantía», un $73 \%$ no reclama por el coste. Disponible en: http://ec.europa.eu/commfrontoffice/publicopinion/archives/ebs/ebs_395_en.pdf acceso de 12 de octubre de 2018.

${ }^{43}$ Ver perfil de consumidor que reclama en: Naomi Creutzfeldt, Project Report: Trusting the middle-man: Impact and legitimacy of ombudsmen in Europe, University of Westminster, junio (2016). Disponible en: https://www.law.ox.ac.uk/sites/files/oxlaw/ ombuds_project_report_nc_2.pdf acceso de 12 de octubre de 2018. 
conoce perfectamente el contenido contractual y se enfrenta a reclamaciones repetitivas, concepto acuñado por los autores anglosajones como «repeat players» ${ }^{44}$.

Finalmente citaremos los problemas especiales que se derivan de la defensa de los intereses difusos, como en la protección de los consumidores y de los derechos medioambientales que formarán parte del segundo estadio que a continuación describiremos. Cappelletti apuntó ${ }^{45}$, con muy buen criterio, que las barreras no pueden ser eliminadas una por una, sino que tendrán que ser eliminadas conjuntamente de forma gradual, ya que la eliminación de una puede agravar otra creando un desequilibrio más perjudicial para el conjunto del sistema.

\subsection{El acceso a la justicia de los consumidores en la Unión Europea}

La evolución natural, tanto a nivel europeo como nacional, ha sido la constitución del acceso a la justicia como Derecho fundamental y, posteriormente, en la segunda generación de Derechos fundamentales ${ }^{46}$ se ha avanzado hacia el reconocimiento del acceso a la justicia de los consumidores en sus reclamaciones de escasa cuantía ${ }^{47}$. En este sentido, y ajustándonos más al reconocimiento de acceso a la justicia de los consumidores dentro del Mercado Único Europeo, observamos dos instrumentos que protagonizan este primer estadio de acceso a la justicia de los consumidores ${ }^{48}$.

Por un lado, la primera apuesta real por parte de la Unión Europea se produjo con el Libro Verde sobre el acceso de los consumidores a la justicia y solución de litigios de consumo en el mercado único de $1993^{49}$. En este documento se sientan las bases y se realiza un estudio comparado de

${ }_{44}$ Término utilizado para referirse el conocimiento adquirido por parte de las grandes compañías o empresarios debido a la experiencia repetida en los procedimientos de reclamación por parte del consumidor en los que ha ejercido la legitimación pasiva, mientras que el consumidor acude en la mayoría de casos por primera vez, sin experiencia alguna sobre el procedimiento al que se va a someter.

${ }^{45}$ Mauro Cappelletti y Bryant G. Garth, «General Report», Access to Justice, Vol I., Book 1, op. cit. p. 54.

46 Francisco José Eguiguren Praeli, «¿Tienen todos los Derechos Humanos igual Jerarquía?», IUS VERITAS, Lima, enero de (1992), pp. 5-6.

47 Nuria Marchal Escalona, «¿Hacia un nuevo derecho procesal europeo de protección del consumidor? La nueva iniciativa europea sobre la resolución de litigios de pequeña cuantía», Revista electrónica de estudios internacionales (REEI), No 28, (2014) p. 7.

48 Aunque como posteriormente analizaremos, existieron movimientos previos a este Libro Verde muy interesantes, sin embargo, he querido poner de relieve los más notorios para explicar estas tres (o cuatro) olas de acceso a la justicia de los consumidores en la UE.

${ }^{49}$ COM (93) 576 final, de 16 de noviembre de 1993. 
los mecanismos judiciales y extrajudiciales aplicables a los litigios de consumo nacionales, así como de asociaciones de consumidores y/o instancias administrativas que ostentaban en diferentes países de la zona euro, las funciones de protección y defensa de los intereses colectivos e individuales de los consumidores.

Esta tarea prosiguió, con el Plan De Acción sobre el acceso de los consumidores a la justicia y la solución de litigios en materia de consumo en el Mercado interior ${ }^{50}$, donde se comenzó a dar respuesta a problemáticas concretas en cuanto a la promoción de los procedimientos extrajudiciales y al acceso de los consumidores a los procedimientos judiciales, amén de establecer unos criterios primigenios para la implantación de procedimientos extrajudiciales aplicables a los litigios en materia de consumo y un formulario tipo de reclamación normalizado para toda Europa. Esta primera aproximación, coincide con el despertar de las conciencias del Estado del bienestar, así como con el crecimiento de las rentas per cápita en las clases medias, que hizo revolucionar el consumo en masa. Sin embargo, quedaban aún muchos retos por batir, tales como la ampliación del cuerpo de abogados de oficio, destinados a las causas de estas personas sin recursos; la ampliación de este servicio social también a las clases medias, pues en ocasiones se cuenta con recursos suficientes para sufragar la asistencia letrada, pero ello mermaría considerablemente la economía familiar, pudiendo entrar en una situación de dificultad económica y, en algunos casos, hasta en riesgo de exclusión social. Y, finalmente, la cuestión que aún sigue sin tener una respuesta eficaz por parte del sistema, las reclamaciones de consumo de escasa cuantía, respecto del coste que supone el proceso de las mismas en comparación con la cantidad reclamada, así como el riesgo a perder el litigio.

2. Segundo estadio de Acceso a la Justicia: representación de los intereses difusos y colectivos de los consumidores

A pesar de que la producción en masa tuvo su auge en los inicios del Siglo XX, tuvimos que esperar décadas para evidenciar la necesidad que plantea la protección de los ciudadanos frente a daños colectivos y la defensa de sus intereses en muchas ocasiones de forma difusa o abstracta ${ }^{51}$. La dificultad de reivindicar estos derechos ante los tribunales de justicia ordinarios tiene su origen en la ardua tarea de definición del grupo de

${ }^{50}$ COM (96) 13 final 14 de febrero de 1996.

${ }^{51}$ Mauro Cappelletti y Bryant G. Garth, «Tendencias en el uso del enfoque de acceso a la justicia». El acceso a la justicia: la tendencia en el movimiento mundial para hacer efectivos los derechos, traducción de Mónica Miranda, Fondo de Cultura Económica, México, (1996), pp. 62-64. 
afectados, por el alto coste que este proceso conlleva, así como el alto riesgo a un fallo negativo o, dicho en otras palabras, el elevado riesgo que debe asumir la parte más débil de este tipo de relaciones jurídicas, principalmente ciudadanos consumidores.

Los procesos colectivos son principalmente aquellos que versan sobre los daños que una o varias empresas han producido a través de un mismo bien o servicio y que este ha afectado por igual a una masa amplia de ciudadanos ${ }^{52}$. Y los procesos en defensa de los intereses difusos son aquellos que han afectado a una masa indeterminada de ciudadanos derivados en su mayoría por delitos consumados contra el medio ambiente ${ }^{53}$. Para ello se debió repensar el papel del proceso civil tradicional ${ }^{54}$, cambiando la concepción individualista de este, por una visión social, plural, de colectividad o grupo actuando conjuntamente por una misma pretensión.

Por un lado, se realizaron tanto reformas legislativas como jurisprudenciales sobre un campo inexplorado hasta el momento. Y, por otro lado, se sentaron también las bases del principio de representación colectiva, en aspectos como la notificación a una sola persona en lugar de a todos los interesados y partes en la causa, ya que esto se convertía en una tarea materialmente casi imposible en los plazos establecidos por los ordenamientos jurídicos de los países miembro para los procesos ordinarios donde solamente existía un demandante y un demandado. Sin embargo, uno de los grandes cambios, a nuestro juicio en el Derecho Procesal Civil, lo constituyó la extensión de la eficacia de los fallos judiciales a todos los afectados, sin la condición de estar personado en la causa. Este último cambio ha sido tratado por multitud de autores como revolucionario, pues cambia el concepto de proceso que se tenía hasta ese momento ${ }^{55}$.

\section{1. Ámbito nacional}

Es necesario apuntar que en España están legislados en estos procesos de acciones colectivas, aunque con mermas importantes sin poder hablar de un

${ }^{52}$ En este sentido ver: Andrea Planchadell Gargallo, Las acciones colectivas en el ordenamiento jurídico español, Tirant Lo Blanch, Valencia, (2014), pp. 63-70.

53 Andrea Planchadell Gargallo, «La consecución de la tutela judicial efectiva en la litigación colectiva», InDret, 4/2015, (octubre 2015), p. 24.

${ }^{54}$ Mauro Cappelletti, «Vindicating the Public Interest Through the Courts: A Comparatist's Contribution, Buffalo L. Rev., 25:643, (1976), pp. 649-690 y Mauro Cappelletti, «La Proteccion D’Interet Collectifs Et De Groupe Dans Le Proces Civil, Metamorphoses De La Procedure Civile», 27, Revue Internationale De Droit Comparé, vol. 27, $\mathrm{n}^{\circ}$ 3, (1975), pp. 571-597.

55 Andrea Planchadell Gargallo, Las acciones colectivas en el ordenamiento jurídico español, op. cit., p. 47. 
proceso colectivo clarificador y sencillo para los ciudadanos. Por lo que no podemos entender como superada en puridad esta barrera por parte del ordenamiento jurídico español. Esta barrera es inferior o más fácilmente superable por los ciudadanos de otros países de la Unión Europea, pero si realmente existe un país referente en lo que a acciones colectivas y procesos por intereses difusos se refiere es Estados Unidos, con sus class actions ${ }^{56}$. También resultan interesante los judicial review emitidos por los Tribunales de Reino Unido para casos de daños colectivos en consumo como los Payment Protection Insurance ${ }^{57}$.

A pesar de no haber conseguido un proceso sencillo para los ciudadanos respecto de las acciones colectivas o de defensa de los intereses difusos, desde hace algunos años se pretende realizar este mismo avance en el campo del arbitraje, sobre todo en el ámbito del consumo, aunque es difícil el encaje de la extensión de la eficacia de los laudos arbitrales a todos aquellos que hayan sido afectados por un mismo proveedor que incurra en una misma infracción, debido al carácter confidencial de cada arbitraje ${ }^{58}$

\section{2. Ámbito Europeo}

Así las cosas, en el entorno europeo, no sería hasta finales de los años noventa cuando se tomará consciencia de la necesidad de perseguir estos intereses difusos ${ }^{59}$ y colectivos, especialmente estos últimos en el ámbito del

${ }^{56}$ Ver más sobre este particular en: Javier López Sanchez, El sistema de las «class actions» en los Estados Unidos de América, Comares, Granada, (2011), pp.11-17; Andrea Planchadell Gargallo, Las acciones colectivas en el ordenamiento jurídico español, op. cit., p. 271-274. y Laura Carballo Piñero, Las acciones colectivas y su eficacia extraterritorial, Universidad de Santiago de Compostela, Santiago de Compostela, 2009, pp. 36-55

57 El Financial Ombudsman requirió este precedente judicial a la Hight Court London en enero de 2011 y desde su web los consumidores de este producto pueden iniciar sus reclamaciones contra los bancos por el cobro de este producto indebido. Disponible en: http://www.financial-ombudsman.org.uk/publications/technical_notes/ppi.html, acceso el día 11 de enero de 2017. Sobre este conflicto, véase Ana Isabel Blanco García, «Conflictos bancarios con consumidores: ¿un mal endémico o un mal común?», Revista Boliviana de Derecho, No. 21, (2016), pp. 242-248.

${ }^{58}$ Ver más sobre este particular en: Ana Montesinos García, «Últimas Tendencias En La Unión Europea Sobre Las Acciones Colectivas De Consumo. La Posible Introducción De Fórmulas De ADR», REDUR 12, diciembre (2014), pp. 87-112.

${ }^{59}$ Los intereses difusos hacen referencia principalmente a grupos de ciudadanos afectados por un daño masivo, que son indeterminables o de difícil determinación. Principalmente daños en el medio ambiente, contaminación ambiental, etc., son muchos los interrogantes que nos suscita esta materia, sin embargo, nos vemos obligados a prescindir de su estudio pues exceden del objeto del presente trabajo. 
consumo ${ }^{60}$ sobre aquellas reclamaciones de escasa cuantía usuales como herramienta para la facilitación del acceso a la justicia.

Como primer ítem hacia una respuesta coordinada e integral en la resolución de conflictos de consumo colectivos podemos señalar la Directiva 98/27EC sobre Reclamaciones Colectivas De Consumo ${ }^{61}$, posteriormente modificada por las nuevas necesidades sociales ${ }^{62}$. Llaman la atención tres documentos publicados en el año 2008, por un lado, las propuestas de acciones colectivas plasmadas en el Libro Blanco de Acciones de daños y perjuicios por incumplimiento de las normas comunitarias de defensa de la competencia ${ }^{63}$ y por otro lado, el Libro Verde sobre el recurso colectivo de los consumidores ${ }^{64} \mathrm{y}$ finalmente la propuesta de consulta pública que plantea el Programa de Trabajo de la Comisión para $2010^{65}$ a través de la Comunicación de la Comisión al Parlamento Europeo, al Consejo, al Comité Económico y Social Europeo y al Comité de las Regiones ${ }^{66}$. Los resultados de estas reformas también fueron respaldados por el Parlamento Europeo con la Resolución del Parlamento Europeo, de 2 de febrero de 2012, «Hacia un planteamiento europeo más coherente del recurso colectivo» ${ }^{67}$, culminando con la Recomendación de la Comisión de 11 de junio de 2013, sobre los principios comunes aplicables a los mecanismos de recurso colectivo de cesación o de indemnización en los Estados miembros en caso de violación de los derechos reconocidos por el Derecho de la Unión ${ }^{68}$.

${ }^{60}$ Reglamento 2006/2004 del Parlamento Europeo y del Consejo, de 27 de octubre de 2004, sobre la cooperación entre las autoridades nacionales encargadas de la aplicación de la legislación de protección de consumidores.

${ }_{61}$ Transpuesta al ordenamiento jurídico español a través de la Ley 39/2002, de 28 de octubre, de transposición al ordenamiento jurídico español de diversas directivas comunitarias en materia de protección de los intereses de los consumidores y usuarios. Esta también regula los intereses difusos de consumidores y usuarios. DOUE núm. 166, de 11 de junio de 1998.

${ }_{62}$ Modificada parcialmente por la Directiva 2002/65/CE del Parlamento Europeo y del Consejo, de 23 de septiembre de 2002, relativa a la comercialización a distancia de servicios financieros destinados a los consumidores, y por la que se modifican la Directiva 90/619/CEE del Consejo y las Directivas 97/7/CE y 98/27/CE. Y más profundamente por la Directiva 2009/22/CE del Parlamento Europeo y del Consejo de 23 de abril de 2009 , relativa a las acciones de cesación en materia de protección de los intereses de los consumidores.

${ }^{63}$ COM (2008) 165 final.

${ }^{64}$ COM (2008) 0794 final.

${ }^{65}$ COM (2010) 608 final.

${ }^{66}$ COM (2010) 135 final, punto 3.1 Construir una Europa de los ciudadanos.

$672011 / 2089$ (INI).

${ }_{68}$ Directiva 2013/396/UE, ampliada por la Directiva 2014/104/UE del Parlamento Europeo y del Consejo de 26 de noviembre de 2014 relativa a determinadas normas por 
Podemos deducir que este segundo estadio de acceso a la justicia a los ciudadanos con intereses colectivos y difusos en la Unión Europea no ha culminado de momento y seguimos teniendo grandes desafíos en este campo. Aunque se ha avanzado desde la normativa europea en las últimas dos décadas, queda camino por recorrer para conseguir una tutela judicial efectiva para que los consumidores puedan actuar de forma coordinada y colectivamente. No obstante, debemos ser optimistas en este sentido y seguir recordando el consejo de Cappelletti, previamente citado, donde nos advertía que las barreras no pueden ser eliminadas una por una, sino que tendrán que ser eliminadas conjuntamente de forma gradual, ya que la eliminación de una puede agravar otra creando un desequilibrio más perjudicial para el conjunto del sistema ${ }^{69}$.

\section{El tercer estadio de Acceso a la Justicia: Resolución Alternativa de conflictos en consumo}

Como tercer escalón del esquema de acceso a la justicia, Cappelletti ${ }^{70}$ estableció en sus estudios el avance necesario hacia la mejora de los mecanismos procesales que no solo faciliten los intereses de los más pobres o de los grupos de intereses difusos o colectivos, sino que también favoreciesen al resto de la población a través de las ventajas que los ADR nos proporcionan, con el correlativo refuerzo por innovar un derecho procedimental más social ${ }^{71}$.

Para la consecución de este propósito Cappelletti argumentó que los ciudadanos precisarían de representación legal ya que de otra manera quedarían sin representación o infrarrepresentados. Sin embargo, se buscó ir más allá de la típica representación legal, administrada en los tribunales de justicia, huyendo de figuras como la fiscalía o los abogados, tanto de oficio como privados, entendiendo una representación sui generis. El foco de atención se sitúa en el amplio abanico de instituciones de ADR -aunque no las nombra como tales en el General Report de su obra- que pueden ser utilizadas por diferentes profesionales y con diferentes procedimientos para resolver e incluso prevenir conflictos en las sociedades modernas.

La Unión Europea, paralelamente a lo teorizado por Cappelletti, una vez quedó garantizado un estándar mínimo de acceso a la justicia de los más desfavorecidos, y encaminadas las legislaciones nacionales para dar la

las que se rigen las acciones por daños en virtud del Derecho nacional, por infracciones del Derecho de la competencia de los Estados miembros y de la Unión Europea.

69 Mauro Cappelletti y Bryant G. Garth, «General Report», Access to Justice, Vol I., Book 1, op. cit. p. 54.

70 Íbidem. pp., 51-53.

${ }^{71}$ Luis Prieto Sanchís, Estudio Sobre Derechos Fundamentales, Ed. Debate, (1990), pp. $215-218$. 
posibilidad de ejercitar las acciones colectivas de los consumidores y usuarios, promueve dar un paso más hacia una tutela efectiva plena. Debemos poner el acento en la necesaria reforma de las leyes procesales como elemento clave para el reconocimiento de estos derechos sustantivos promulgados. En este sentido debemos advertir que a pesar de la pluralidad de normas promulgadas por la Unión Europea donde se amplían los derechos sustantivos de los consumidores, existe muy poca eficacia práctica de las mismas cuando estas tienen que ser exigibles por los ciudadanos ante los Tribunales de justicia.

Es ahí donde surge la necesidad de establecer los cambios necesarios en la legislación procesal para que reconozcan a los ADR como posibles enjuiciadores de estos conflictos, a fin de otorgar una tutela efectiva de los derechos de los consumidores. Este desiderátum podría ser llevado a cabo mediante un re-pensamiento del sistema judicial, de modo que se puedan suplir, con coherencia, a los tribunales de justicia en aquellos casos donde la complejidad del conflicto sea menor y ante materias principalmente económicas.

Esta apertura del acceso a la justicia, analizada anteriormente, podría llevar aparejada una previsión de intensificación en las demandas de las comunidades con escasez de recursos y los grupos con intereses difusos y colectivos con dificultades en el acceso a la tutela judicial efectiva. Por ello, se deben plantear cambios en los procesos, en la estructura judicial, proyectar nuevos tribunales, incluir profesionales expertos en los procedimientos ADR, así como dar cabida a los colegios de abogados para llevar a cabo este tipo de procedimientos. No obstante, estos cambios procesales exigen otros cambios en el Derecho sustantivo, con la finalidad de evitar conflictos y facilitar la resolución de los mismos de forma más privada e informal. En este momento es importante buscar nuevas fórmulas procesales como las que ofrece el sistema The multi-doors-Justice System o Multi-rooms Justice System, en la que las ADR y la Jurisdicción coexisten como mecanismos complementarios de tutela de los ciudadanos ${ }^{72}$, incluyendo tanto medios out of Court como in Court $^{73}$. Estos mecanismos ya están siendo aplicados en el sistema estadounidense con gran éxito tanto en tribunales ordinarios ${ }^{74}$, como en grandes firmas de despachos de abogados. Así las cosas, los estadounidenses llevan décadas

${ }^{72}$ Concepto creado en el año 1976 por el Profesor Frank E. A. Sander de la Harvard Law School y desarrollado años después por la doctrina. Larry Ray y Anne Clare, «The Multi-Door Courthouse Idea: Building the Courthouse of the Future...Today», Ohio State Journal on Dispute Resolution, Vol. 1, Issue 1, (1985), pp. 9-12.

73 Íbidem, p. 39

74 Tribunales del distrito de Columbia y proyectos aislados en Houston, Tulsa y Washington, D.C. ver más ejemplos de experiencias en: James Podgers «Multi-door proposal reflects growing role of ADR», ABA Journal, (septiembre 1993), p. 188 y Kenneth K. Stuart y Cynthia A.Savage, «Multi-Door Courthouse: How It's Working», The Colorado Lawyer, Vol. 26, Issue 10, (October 1997), pp. 13-18. 
estudiando y poniendo en práctica este sistema también llamado multi-doors courthouse $^{75}$. En definitiva, es un sistema que inicia su mecanismo con un diagnóstico del conflicto y prosigue con la consecuente prescripción del medio de resolución adecuado al mismo.

Partimos hacia la búsqueda de este concepto de justicia integral, conocedores de la «door» judicial tradicional para las reclamaciones de los consumidores - tanto individuales como colectivas-, pero con expectativas ambiciosas en las «doors» que el ADR y el ODR nos abren para la resolución de los conflictos de consumo de una manera más sencilla, pacifica, rápida y de calidad, adaptada a nuestras necesidades, no ya como consumidores, sino como ciudadanos con derecho a que no sean menoscabados nuestros legítimos intereses económicos recogidos en el artículo $51 \mathrm{CE}$.

Por otro lado, también se ha planteado por la doctrina la necesidad de adaptar el proceso civil al tipo de disputa, en función de diferentes ítems, ya que conflictos simples o de escasa cuantía pueden colapsar el sistema y dilatar otros de mayor enjundia, amén de que la naturaleza de ciertos conflictos bloquea transacciones comerciales hasta la resolución del conflicto. Para ello, debemos tener en cuenta si el conflicto proviene de una relación puntal o de una relación contractual de medio-largo plazo, así como las capacidades de las partes para llegar a un acuerdo común ${ }^{76}$.

Si seguimos a Cappelletti, quien con buen criterio apuntó claramente a las ADR como la última pieza de la pirámide hacia el acceso a la justicia, podemos analizar también paralelamente la trayectoria con la seguida por la Unión Europea en la utilización de estas técnicas como mecanismos que facilitan el acceso a la justicia, haciéndola más rápida y eficaz. Esto comenzó a ser una realidad a través de la Directiva 2008/52 del Parlamento Europeo y del Consejo, de 21 de mayo de 2008, sobre ciertos aspectos de la mediación en asuntos civiles y mercantiles $^{77}$ y la Directiva 2013/11/UE del Parlamento Europeo y del Consejo, de 21 de mayo de 2013, relativa a la resolución alternativa de litigios en materia de consumo. Con ellas se ha pretendido otorgar y ampliar garantías y derechos a los ciudadanos más allá de si éstos actúan colectiva o individualmente en su posición de consumidor, pero sobre todo en su posición de personas consumidoras. No obstante, existen infinidad de críticas que estos sistemas nos

${ }^{75}$ Larry Ray y Anne Clare, «The Multi-Door Courthouse Idea: Building the Courthouse of the Future...Today», Ohio State Journal on Dispute Resolution, op. cit, (1985), p. 11.

${ }^{76}$ Austin Sarat, «Alternatives in Dispute Processing: Litigation in a Small Claims Court», Law \& Society Review, Vol. 10, Issue 3 (Spring 1976), pp. 341-378.

${ }_{77}$ Inicialmente por un error de traducción de la Directiva original a la versión española, España transpuso esta Directiva prohibiendo incluir dentro del ámbito de aplicación de la norma la mediación de consumo. Afortunadamente desde la Ley 7/2017 se ha eliminado esta prohibición. 
despiertan a la hora de su aplicación sobre todo los que afectan al ámbito de las garantías procesales, donde debemos extremar las precauciones para que esta justicia alternativa no se convierta en una justicia de «saldo».

\section{Cuarto Estadio de Acceso a la Justicia: Resolución Alternativa de Conflictos de consumo en Linea}

En la presente era, donde nuestras vidas son influenciadas por las nuevas tecnologías de la información y las telecomunicaciones, nos ha parecido oportuno avanzar hacia un punto inconcebible para la doctrina hace tan solo unas décadas en cualquier estudio transversal sobre acceso a la justicia con dimensión social y constitucional. Así las cosas, autores como Cappelletti estarían hoy aprovechando esta nueva era digital para invitar a mejorar el acceso a la justicia a través del Online Dispute Resolution y teorizando quizás con la cuarta «ola» de acceso a la justicia a través del ODR.

Sin embargo, la vocación para el desarrollo del ODR mundial tiene más aspiraciones mercantilistas y economicistas que humanas o sociales. Todo ello está derivando en un sistema donde la propensión principal del ODR no es el acceso a la justicia (social) para los ciudadanos de una forma más fácil, rápida y económica $^{78}$, sino la creación de un elemento para generar confianza ${ }^{79}$ a los consumidores como agentes potenciales de consumo. De este modo se desvirtúa la concepción del ciudadano con derechos inherentes que hasta este momento nos ha acompañado y se intercambia por la concepción de consumidor como agente que realiza transacciones económicas dentro de un mercado común ${ }^{80}$.

En esta línea, la Unión Europea puso en marcha -en febrero de 2015- la plataforma de ODR de consumo desarrollada a través del Reglamento 524/2013 del Parlamento Europeo y del Consejo, de 21 de mayo de 2013, sobre resolución de litigios en línea en materia de consumo ${ }^{81}$. A través de esta plataforma ${ }^{82}$ se permite a ciudadanos de cualquier país miembro realizar reclamaciones de consumo a empresas sitas en cualquier país de la Unión Europea con la

${ }_{78}$ Report on the Application of the EU Charter of Fundamental Rights 2015, European Union, (2016).

79 Inmaculada Barral Viñals, «La mediación y el arbitraje de consumo: explorando sistemas de ODR», Revista de Internet, Derecho y Política, No 11, (2010), p. 7-8.

${ }^{80}$ Siguiendo a Silvia Barona Vilar, ««Justicia integral» y «Acces to Justice». Crisis y evolución del «paradigma»»», en Mediación, Arbitraje y Jurisdicción en el actual paradigma de Justicia, coord. por Silvia Barona Vilar, Thomson Reuters-Civitas, Navarra, (2016), pp. 45-46.

${ }^{81}$ y por el que se modifica el Reglamento (CE) no 2006/2004 y la Directiva 2009/22/ CE, DOC 181 de 21 de junio de 2012, p. 99. DOUE núm. 165, de 18 de junio de 2013.

${ }^{82}$ Disponible en: https://ec.europa.eu/consumers/odr/main/index.cfm?event=main. home2.show\&lng=ES, acceso el día 24 de octubre de 2018. 
finalidad de crear un verdadero Mercado Común. Sin embargo, son muchos los retos que le quedan por cumplir a esta herramienta y a los Estados participantes de la misma, como mejorar la inversión dedicada a estos medios, que figura como el ítem más infra financiado dentro de los ítems que sirven de apoyo y desarrollo de la justicia según el ultimo índice sobre la justicia de la Unión Europea $^{83}$.

El ODR esta llamado a ser un elemento necesario para el progreso y la confianza $^{84}$ en el ADR de la Unión Europea, más aún si el campo donde se aplica es el consumo. Sin embargo, para llevar a cabo tal empresa deberemos darles a los ciudadanos la oportunidad de expresarse por sí mismos, hacerlos sentirse escuchados para fomentar su confianza en el sistema, proporcionarles un foro neutral y tratarlos con el suficiente respeto ${ }^{85}$. Estos principios no son nuevos, pero deben ser actualizados para que el ODR se convierta en una herramienta eficaz y de futuro para la defensa de los intereses individuales y colectivos de la sociedad actual.

No obstante, este sistema es contemplado como si de un lujo se tratase debido a la pretensión de dar una alta calidad de justicia las partes cuando estas no puedan superar las barreras estructurales para hacer realidad sus derechos ante una tipología amplia de reclamaciones.

El acceso a la justicia trata de combatir las barreras planteadas de forma integral, cuestionando el conjunto completo de instituciones, procedimientos, procesos y recursos humanos que caracterizan a nuestros sistemas judiciales. El único riesgo al que nos enfrentamos, y no por ello banal, es que el uso de procedimientos rápidos y la utilización de personal de bajo coste puedan producir un resultado o producto de justicia de segunda categoría. La tarea procesalista en este ámbito se debe basar en aportar mejoras en la seguridad jurídica y en la tutela judicial efectiva a la que las personas consumidoras tienen derecho. En esta reflexión sobre las reformas normativas se deben tener en cuenta los límites y la capacidad de los tribunales, de los procedimientos y de los juristas. El objetivo no es crear una justicia más «pobre», sino hacerla más accesible para todos ${ }^{86}$.

${ }^{83}$ Figura 2 y Figura 3, The 2018 EU Justice Scoreboard, pp. 15 y 16 disponible en: https://ec.europa.eu/info/sites/info/files/justice_scoreboard_2018_en.pdf, acceso el día 11 de octubre de 2018.

${ }^{84}$ Así lo ha entendido Reino Unido con su iniciativa de reforma del proceso civil a través del proyecto: «Online Dispute Resolution For Low Value Civil Claims», de febrero de 2015, disponible en: https:/www.judiciary.gov.uk/reviews/online-dispute-resolution/ odr-report-february-2015/ acceso el 12 de octubre de 2018.

${ }^{85}$ Tom R. Tyler., Why People Obey the Law, Yale University Press, Michigan, (1990), pp. 115-160.

${ }^{86}$ Mauro Cappelletti y Bryant G. Garth., Access to Justice, op. cit. p. 122. 


\title{
EL DERECHO FUNDAMENTAL DE ACCESO A LA JUSTICIA DE LOS CONSUMIDORES
}

\author{
The Fundamental Right of Access \\ to Justice for Consumers
}

\author{
María José Catalán Chamorro \\ Universidad de Valencia (España) \\ maria.jose.catalan@uv.es
}

http://dx.doi.org/10.18543/ed-66(2)-2018pp323-346

\section{Copyright}

Estudios de Deusto es una revista de acceso abierto, lo que significa que es de libre acceso en su integridad. Se permite su lectura, la búsqueda, descarga, distribución y reutilización legal en cualquier tipo de soporte sólo para fines no comerciales, sin la previa autorización del editor o el autor, siempre que la obra original sea debidamente citada y cualquier cambio en el original esté claramente indicado 